\title{
Structural Analyses of the SIm1-PH Domain Demonstrate Ligand Binding in the Non-Canonical Site
}

\author{
Kanchan Anand ${ }^{1,2 *}$, Kenji Maeda ${ }^{1}$, Anne-Claude Gavin ${ }^{1 *}$
}

1 European Molecular Biology Laboratory Heidelberg, Structural and Computational Biology Unit, Heidelberg, Germany, 2 Max-Planck-Institut für Molekulare Physiologie, Abteilung Physikalische Biochemie, Dortmund, Germany

\begin{abstract}
Background: Pleckstrin homology $(\mathrm{PH})$ domains are common membrane-targeting modules and their best characterized ligands are a set of important signaling lipids that include phosphatidylinositol phosphates (PtdlnsPs). PH domains recognize PtdlnsPs through two distinct mechanisms that use different binding pockets on opposite sides of the $\beta$ strands 1 and 2 : i) a canonical binding site delimited by the $\beta 1-\beta 2$ and $\beta 3-\beta 4$ loops and ii) a non-canonical binding site bordered by the $\beta 1-\beta 2$ and $\beta 5$ - $\beta 6$ loops. The $\mathrm{PH}$ domain-containing protein SIm1 from budding yeast Saccharomyces cerevisiae is required for actin cytoskeleton polarization and cell growth. We recently reported that this PH domain binds PtdlnsPs and phosphorylated sphingolipids in a cooperative manner.

Principal Findings: To study the structural basis for the Slm1-PH domain (SIm1-PH) specificity, we co-crystallized this domain with different soluble compounds that have structures analogous to anionic lipid head groups of reported SIm1 ligands: inositol 4-phosphate, which mimics phosphatidylinositol-4-phosphate (Ptdlns(4)P), and phosphoserine as a surrogate for dihydrosphingosine 1-phosphate (DHS1-P). We found electron densities for the ligands within the socalled non-canonical binding site. An additional positively charged surface that contacts a phosphate group was identified next to the canonical binding site.

Conclusions: Our results suggest that SIm1-PH utilizes a non-canonical binding site to bind PtdlnsPs, similar to that described for the PH domains of $\beta$-spectrin, Tiam1 and ArhGAP9. Additionally, SIm1-PH may have retained an active canonical site. We propose that the presence of both a canonical and a non-canonical binding pocket in SIm1-PH may account for the cooperative binding to PtdlnsPs and DHS-1P.
\end{abstract}

Citation: Anand K, Maeda K, Gavin A-C (2012) Structural Analyses of the SIm1-PH Domain Demonstrate Ligand Binding in the Non-Canonical Site. PLoS ONE 7(5): e36526. doi:10.1371/journal.pone.0036526

Editor: Beata G. Vertessy, Institute of Enzymology of the Hungarian Academy of Science, Hungary

Received October 29, 2011; Accepted April 2, 2012; Published May 4, 2012

Copyright: (c) 2012 Anand et al. This is an open-access article distributed under the terms of the Creative Commons Attribution License, which permits unrestricted use, distribution, and reproduction in any medium, provided the original author and source are credited.

Funding: This work was partially funded by the Federal Ministry of Education and Research (BMBF; FKZ0315162) in the framework of the BioChancePLUS-4 to ACG. No additional external funding received for this study. The funders had no role in study design, data collection and analysis, decision to publish, or preparation of the manuscript.

Competing Interests: The authors have declared that no competing interests exist.

* E-mail: gavin@embl.de (ACG); anand@embl.de (KA)

\section{Introduction}

Pleckstrin homology $(\mathrm{PH})$ domains are short segments of approximately 120 amino acids and are present in a wide range of proteins. Despite their poor conservation at the sequence level, $\mathrm{PH}$ domains share a common fold that consists of a seven-stranded antiparallel $\beta$-sheet and a C-terminal amphipathic $\alpha$-helix (Figure 1A). The human genome and that of the yeast Saccharomyces cerevisiae encode at least 252 and 27 proteins, respectively, that contain one or several $\mathrm{PH}$ domains and these are involved in a wide range of biological processes, including cellular signaling, cytoskeleton organization, membrane trafficking and lipid metabolism $[1,2]$.

The best-characterized ligands for $\mathrm{PH}$ domains are phosphatidylinositol phosphates (PtdInsPs) [1,3,4,5,6,7,8,9,10,11] and binding to this important class of signaling lipid contributes to protein recruitment to cellular membranes. The ligand specificity of each $\mathrm{PH}$ domain is variable. For example, the $\mathrm{PH}$ domain of phospholipase $\mathrm{C} \delta$ (PLC $\delta$ ) binds phosphatidylinositol-4,5-bisphosphates $\left(\operatorname{Ptd} \operatorname{Ins}(4,5) \mathrm{P}_{2}\right)$ with high affinity $\left(\mathrm{K}_{\mathrm{D}}=2 \mu \mathrm{M}[1]\right)$, whereas the affinity of the dynamin $1 \mathrm{PH}$ domain for the same phospholipid is in the millimolar range. The protein kinase $\mathrm{B}$ (b/akt) $\mathrm{PH}$ domain, on the other hand, binds $\operatorname{PtdIns}(3,4,5) \mathrm{P}_{3}$ and PtdIns(3,4) $\mathrm{P}_{2}$ with similar affinities, but does not bind to PtdIns(3)P or $\operatorname{PtdIns}(3,5) \mathrm{P}_{2}$. However, only a minority of $\mathrm{PH}$ domains actually target PtdInsPs with high specificities and affinities and in fact, the vast majority of $\mathrm{PH}$ domains either poorly discriminate between different PtdInsPs or do not bind lipids at all $[12,13,14,15]$. Accordingly, some PH domains have been proposed to mediate protein-protein interactions or to bind proteins and lipids through cooperative mechanisms [16].

Both X-ray crystallography and nuclear magnetic resonance spectroscopy (NMR) have been used to understand the structural determinants of lipid recognition by $\mathrm{PH}$ domains. In general, head groups of PtdInsPs are accommodated on a positively charged surface of $\mathrm{PH}$ domains and two main binding modes have been described to date (Figure 1A). First, a so-called canonical binding site has been characterized that uses positively charged residues located between the $\beta 1-\beta 2, \beta 3-\beta 4$ and $\beta 6-\beta 7$ loops. Canonical binding sites have been reported for a variety of $\mathrm{PH}$ domains, such 

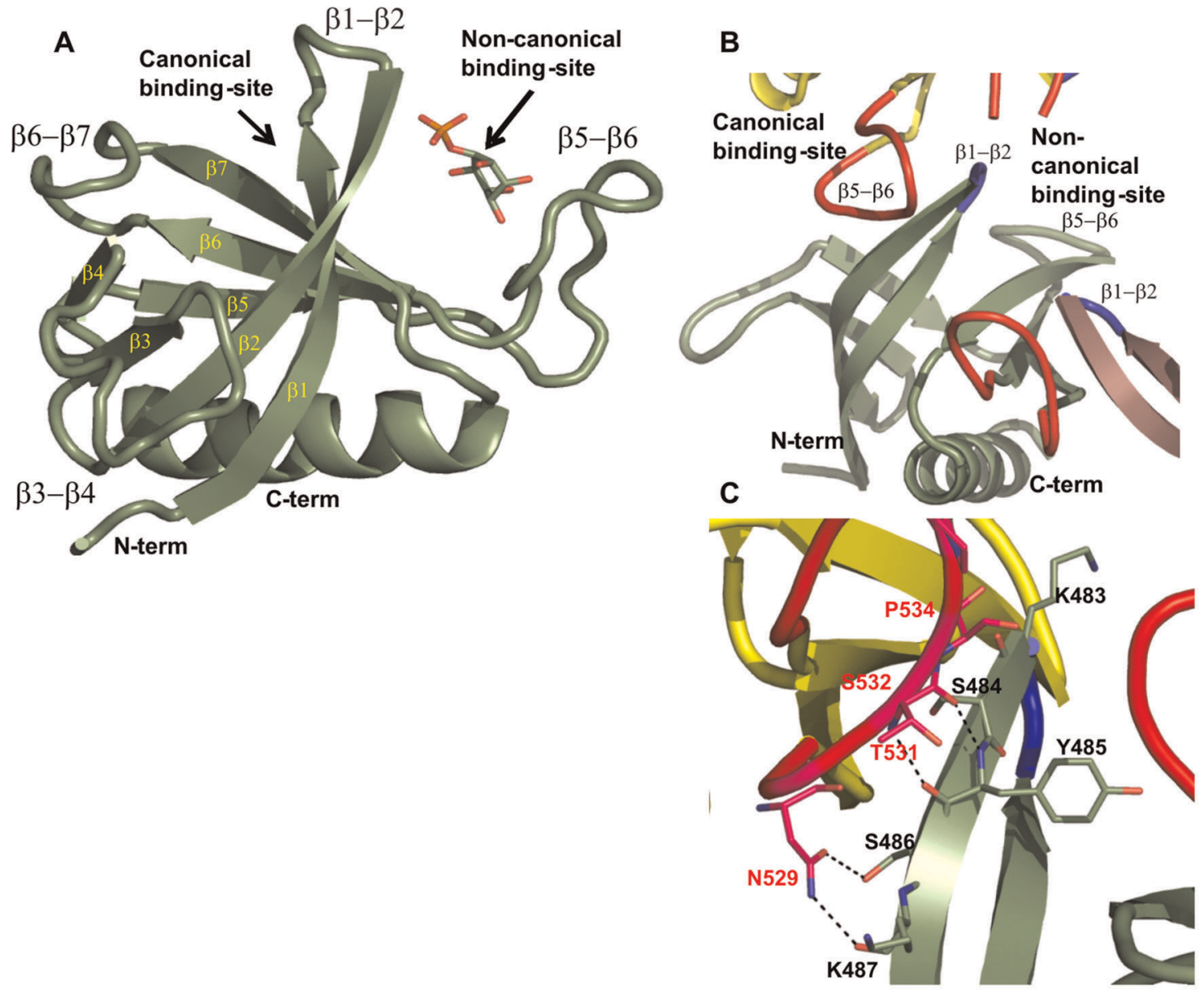

Figure 1. Overall structure of the SIm1-PH domain. (A) Ribbon representation of the $1.76 \AA$ structure (green) showing that SIm1-PH folds into a seven-stranded $\beta$-sheet terminated with an $\alpha$-helix. All secondary structure elements are labeled in yellow and black. Both canonical and noncanonical binding sites are also marked. (B) In the X-ray crystal structure the canonical binding site is partially occluded by the $\beta 5-\beta 6$ loop (in red) of the neighboring molecules (in yellow and violet) on both sides. The $\beta 1-\beta 2$ loops of all the molecules are shown in blue. All the loops in Figure $1 \mathrm{~B}$ have been smoothened using PyMOL for clarity. (C) Detailed view of the interactions between the $\beta 1-\beta 2$ loop and the $\beta 5-\beta 6$ loop of neighboring molecules. Primarily, the side-chain residues of the $\beta 5-\beta 6$ loop are making contact with the main-chain atoms of the $\beta 1-\beta 2$ loop region. doi:10.1371/journal.pone.0036526.g001

as those of PLC $\delta$ and b/akt [6,8]. Second, a non-canonical binding site has been observed for the $\mathrm{PH}$ domain of $\beta$-spectrin, Tiaml and ArhGAP9 that is formed between the $\beta 1-\beta 2$ and $\beta 5-\beta 6$ loops, adjacent to the canonical site but on the other side of the $\beta 1$ $\beta 2$ loop $[17,18,19]$.

The PH domain-containing protein Slm1 from S. cerevisiae is a component of the target of rapamycin complex 2 (TORC2) signaling pathway. Slml plays a role in the regulation of actin organization [20] and is also a target of sphingolipid signaling during the heat shock response [10]. The PH domain of Slml is known to bind to $\operatorname{PtdIns}(4,5) \mathrm{P}_{2}$ [20], and also $\operatorname{PtdIns}(4) \mathrm{P}$ and a phosphorylated sphingolipid species, dihydrosphingosine 1phosphate (DHS-1P) [12] (Figure S1). We have previously reported that in vitro, the Slm1-PH domain (Slm1-PH) interacts with liposomes containing $\operatorname{PtdIns}(4,5) \mathrm{P}_{2}$ and DHS-1P cooperatively. Similarly, in vivo Slm 1 association with specialized membrane microdomains, the eisosomes, requires the metabolism of both PtdIns $(4,5) \mathrm{P}_{2}$ and sphingolipids [12]. This led to the view that the recruitment of Slm1-PH to cellular membranes might involve a novel mechanism, in which not only PtdInsPs but also phosphorylated sphingolipids could act as anchoring ligands. We previously reported a structure for the apo $\operatorname{Slm} 1-\mathrm{PH}($ at $2.0 \AA$ ) that depicted only part of the non-canonical binding site. This revealed that Slm1-PH might possess two binding sites for anionic lipids [12]. However, in the absence of a ligand-bound holo structure, the detailed modalities of ligand recognition remained largely elusive.

Here we report the crystal structure of the apo form of Slm 1-PH domain at $1.76 \AA$ resolution together with the holo Slm1-PH bound to different mimics of lipid head groups: i) a $1.68 \AA$ resolution structure of Slm1-PH in complex with phosphoserine (surrogate of DHS-1P head group) and ii) $1.4 \AA$ and $1.8 \AA$ resolution structures of Slm1-PH in complex with inositol 4phosphate $(\operatorname{Ins}(4) \mathrm{P}$; surrogate of the $\operatorname{PtdIns}(4) \mathrm{P}$ head group). The results show that the non-canonical binding site of Slm1-PH is used for ligand binding, similar to the site described previously for $\beta$-spectrin, Tiam1 and ArhGAP9 [17,18,21] and which therefore suggests that this interaction mode has been conserved in yeast. Furthermore, our data also support the existence of an active canonical binding site in Slm1-PH, thus providing a structural model for the previously reported cooperativity between PtdInsPs and DHS-1P binding [12]. 


\section{Results and Discussion}

\section{Ligand screening for the SIm1-PH domain}

Previous experiments, based on isothermal titration calorimetry (ITC), have shown that Slm1-PH binds weakly to $\operatorname{PtdIns}(4,5) \mathrm{P}_{2}$ or DHS-1P alone. Both lipids need to be present for the interaction to reach affinities of $1.8 \mu \mathrm{M}$ [12], which is similar to those observed for other $\mathrm{PH}$ domain-ligand pairs such as PLC $\delta$-PH and $\operatorname{PtdIns}(4,5) \mathrm{P}_{2} \quad(1.7 \mu \mathrm{M} ; \quad[6])$ or $\beta$-spectrin and $\operatorname{PtdIns}(4,5) \mathrm{P}_{2}$ (40 $\mu \mathrm{M}$; [18]). To understand the interactions and specificities of Slm1-PH in complex with surrogate lipid head groups, more than 50 crystallization experiments were screened that included both co-crystallization and soaking techniques. Seven different soluble compounds that mimic PtdInsPs and DHS-1P head groups (Figure S1) were used either alone or in combination. These included various inositol phosphates (InsPs), phosphoserine, the sphingosine analog FTY720 and its phosphorylated derivative, FTY720(P) $[22,23,24]$ (Table 1). Only co-crystallization experiments with bound ligands produced acceptable diffraction data. The results presented here include: (i) the apo structure of Slm1$\mathrm{PH}$ that we resolved at $1.76 \AA$ resolution; (ii) one structure with phosphoserine at $1.68 \AA$ resolution; and (iii) two structures of Slml-PH in complex with $\operatorname{Ins}(4) \mathrm{P}$ at 1.4 and $1.8 \AA$ resolution (Tables 1 and 2).

\section{Overall structure of the SIm1-PH domain}

Although the sequence identity with other $\mathrm{PH}$ domains is rather low $(\sim 18 \%)$, the overall fold of Slm1-PH is similar to previously determined structures of $\mathrm{PH}$ domains [6,16,17,18,25] (Figure 1A). The core of each monomer consists of seven antiparallel $\beta$-strands with a C-terminal amphipathic $\alpha$-helix connected via a long and flexible loop. The loops connecting the $\beta$-sheets, especially $\beta 3-\beta 4$ and $\beta 5-\beta 6$ (residues 501-512 and residues 526-542, respectively), are long and constitute putative ligand-binding sites. The interior of the core consists of hydrophobic residues. Electron densities corresponding to the Ins(4)P molecules were visible between the $\beta 1-\beta 2$ and $\beta 5-\beta 6$ loop regions (difference density maps; Figure S2). In addition, all structures in complex with ligands show regions of additional density between the bottom of the $\beta 1-\beta 2$ loop and the N-terminus of Slm1-PH. Intriguingly, we could not assign any ligand to these extra densities. Interestingly for all four structures, much of the binding space in the canonical binding site is occupied by the $\beta 5-\beta 6$ loop of the neighboring molecule (Figure $1 \mathrm{~B}$ and $\mathrm{C}$ ). As the interactions between the two molecules involve the backbone of $\beta 1-\beta 2$ and no specific side-chain residue, this probably represents a crystallographic packing artifact. Compared with our previously published structure at $2 \AA$ [12], our new apo structure clearly visualizes the full $\beta 5-\beta 6$ loop, which plays a key role in accommodating ligand molecules in the noncanonical binding site (see below).

\section{The SIm1-PH domain binds lipid via a non-canonical binding site}

In our complex crystal structures, all negatively charged ligands localize to the non-canonical binding site defined by the flexible loops $\beta 1-\beta 2$ and $\beta 5-\beta 6$, which form a wide and shallow pocket (Figure 1A). As described for other PH domains, the $\beta 1-\beta 2$ sheets and their connecting loop play a central role in ligand binding (Figure 2) $[2,5,8,11,16,17,18,26,27]$. For the two structures determined in complex with $\operatorname{Ins}(4) \mathrm{P}$, the positions of the inositol ring overlap. In both cases, the oxygen atoms of the inositol ring contact the $\beta 1-\beta 2$ region through the hydroxyl group of Tyr485 (hydrogen bond length $=2.8 \AA$ ) and the $\mathrm{NH}_{2}$ group of Arg478 (hydrogen bond length $=2.5 \AA$ ), and the $\beta 5$ - $\beta 6$ loop through the

Table 1. Crystallographic screening for SIm1-PH domain ligands.

\begin{tabular}{|c|c|c|}
\hline Surrogate ligands & Result & Lipid \\
\hline Apo SIm1-PH & $1.76 \AA ̊$ resolution & Holo form \\
\hline Ins(4)P & $1.4 \AA$ A resolution; Ins(4)P bound & PtdIns(4)P \\
\hline Ins(4)P & $1.8 \AA$ A resolution; Ins(4)P bound & Ptdlns(4)P \\
\hline PS+Ins $(1,4,5) P_{3}$ & 1.68 Å resolution; Phosphoserine bound & DHS-P + PtdIns $(4,5) P_{2}$ \\
\hline$P S+\operatorname{lns}(4) P$ & $2.7 \AA$ resolution; Phosphoserine bound & DHS-1P + Ptdlns(4)P \\
\hline FTY720(P)+Ins $(4,5) P_{2}$ & $1.6 \AA$ resolution; No bound ligand & DHS-1P + Ptdlns $(4,5) P_{2}$ \\
\hline $\operatorname{lns}(3) P$ & $1.7 \AA$ resolution; No bound ligand & Ptdlns(3)P \\
\hline PS+Ins (3)P & $1.6-1.8 \AA$ resolution; No bound ligand & DHS-1P + Ptdlns(3)P \\
\hline$P S+\operatorname{lns}(4,5) P_{2}$ & $2.0 \AA$ resolution; No bound ligand & DHS-1P + Ptdlns $(4,5) P_{2}$ \\
\hline$P S+\operatorname{lns}(1,4,5) P_{3}$ & $2.5 \AA$ resolution; No bound ligand & DHS-1P + Ptdlns $(4,5) \mathrm{P}_{2}$ \\
\hline $\operatorname{lns}(1,4,5) \mathrm{P}_{3}$ & $2.5 \AA$ resolution; but only in one direction & Ptdlns $(4,5) \mathrm{P}_{2}$ \\
\hline $\operatorname{lns}(1,4,5) \mathrm{P}_{3}$ & $2.6 \AA$ resolution; No bound ligand & Ptdlns $(4,5) \mathrm{P}_{2}$ \\
\hline$P S+\operatorname{lns}(1,4,5) P_{3}$ & $3.1 \AA$ resolution; No bound ligand & DHS-1P + Ptdlns $(4,5) P_{2}$ \\
\hline $\operatorname{lns}(4,5) \mathrm{P}_{2}$ & $3.4 \AA$ A resolution; No bound ligand & Ptdlns $(4,5) \mathrm{P}_{2}$ \\
\hline$P S+\operatorname{lns}(1,4,5) P_{3}$ & $4.5 \AA$ resolution; No bound ligand & DHS-1P + Ptdlns $(4,5) P_{2}$ \\
\hline FTY720 & Too mosaic diffraction to process so far & DHS \\
\hline $\operatorname{lns}(4) P+\operatorname{lns}(1,4,5) P_{3}$ & No diffraction & Ptdlns(4)P+Ptdlns $(4,5) P_{2}$ \\
\hline FTY720(P)+Ins(4)P & No crystals & DHS-1P + Ptdlns(4)P \\
\hline
\end{tabular}

*PS = phosphoserine.

${ }^{*}$ Crystals of the same protein-ligand complex were obtained in different crystallization conditions.

${ }^{*}$ FTY720 = synthetic analog of sphingosine, currently studied as a potent immunosuppressive and immunomodulatory agent [22,23,24]; FTY720(P) = phophorylated FTY720.

doi:10.1371/journal.pone.0036526.t001 
Table 2. Data collection and refinement statistics.

\begin{tabular}{|c|c|c|c|c|}
\hline & Apo & $\begin{array}{l}\text { Phosphoserine and } \\
\text { malonate }\end{array}$ & Ins(4)P & $\begin{array}{l}\text { Ins(4)P (inverted phosphate } \\
\text { group) }\end{array}$ \\
\hline X-ray source & Synchrotron $^{\mathrm{a}}$ & Synchrotron $^{b}$ & Synchrotron $^{\mathrm{a}}$ & Synchrotron $^{a}$ \\
\hline Detector & ADSC Q105 CCD @ ESRF & MAR225 @ SLS & ADSC Q105 CCD @ ESRF & ADSC Q105 CCD @ ESRF \\
\hline Wavelength $(\AA)$ & 0.97242 & 1.0000 & 1.06650 & 1.06650 \\
\hline Temperature (K) & 100 & 100 & 100 & 100 \\
\hline $\begin{array}{l}\text { Resolution }(\AA) \text { at which data } \\
\text { were collected }\end{array}$ & 1.60 & 1.60 & 1.40 & 1.75 \\
\hline \multicolumn{5}{|c|}{ Data Statistics for refined atoms } \\
\hline Space group & P21 & P22121 & P21 & P21 \\
\hline No. of molecules in a.u. & 4 & 2 & 4 & 4 \\
\hline Unit cell dimensions & $37.3,82.3,76.4, \beta=90.1$ & $37.5,73.6 .82 .5, \beta=90.01$ & 37.0,71.6.82.7, $\beta=90.02$ & $37.0,71.6 .82 .6, \beta=90.1$ \\
\hline $\mathrm{R}_{\text {meas }}{ }^{\mathrm{c}}(\%)$ & $4.4(30.0)$ & $9.7(53.5)$ & $4.5(53.2)$ & $5.0(35.0)$ \\
\hline $1 / \sigma$ & $26.02(5.0)$ & $10.6(3.3)$ & $16.6(2.5)$ & $18.7(4.0)$ \\
\hline Completeness (\%) & $95.6(70.3)$ & $98.6(93.3)$ & $95.6(92.8)$ & $99.2(96.0)$ \\
\hline Redundancy & $6.6(4.0)$ & $5.3(6.9)$ & $4.6(3.6)$ & $3.7(3.5)$ \\
\hline Unique reflections & 50454 & 26485 & 80522 & 39703 \\
\hline No. of reflections used & 43737 & 26460 & 73073 & 37717 \\
\hline \multicolumn{5}{|l|}{ Refinement } \\
\hline Resolution (Å) & 1.76 & 1.68 & 1.45 & 1.80 \\
\hline$R_{\text {work, }} R_{\text {free }}{ }^{d}$ & $18.8 / 22.7$ & $21.4 / 24.1$ & 19.2/22.6 & $18.6 / 24.0$ \\
\hline \multicolumn{5}{|l|}{ No. of atoms } \\
\hline Protein & 3692 & 1739 & 3500 & 3543 \\
\hline Water & 357 & 180 & 380 & 273 \\
\hline Ligand(s) & 10 & 31 & 88 & 102 \\
\hline \multicolumn{5}{|c|}{ B factors [average B value $\left(\AA^{2}\right)$ for the refined atoms] } \\
\hline Protein & 27.5 & 31.3 & 23.0 & 21.7 \\
\hline Water & 35.9 & 37.9 & 36.5 & 33.2 \\
\hline Ligand(s) & 61.2 & 85.8 & 67.6 & 69.3 \\
\hline \multicolumn{5}{|l|}{ R.m.s deviations } \\
\hline Bond lengths $(\AA)$ & 0.006 & 0.006 & 0.006 & 0.011 \\
\hline Bond angles $\left({ }^{\circ}\right.$ ) & 1.04 & 1.11 & 1.02 & 1.83 \\
\hline
\end{tabular}

*Highest resolution shell is shown in parenthesis.

abeam line ID29 at European Synchrotron Radiation Facility at Grenoble in France.

${ }^{b}$ PX beam line at Swiss Light Source, Paul Scherrer Institut 5232 Villigen PSI Switzerland.

${ }^{\mathrm{C}} \mathrm{Rmeas}$ is the redundancy-independent merging R-factor (intensities) Rmeas $=\left(\Sigma h(n /(n-1))^{0.5} \Sigma j|\hat{l} h-l h j|\right) /(\Sigma h j l h j)$ with $\hat{l} h=(\Sigma j l h j) / n j$ Where $N$ is the number of times a given observation has been observed (ie $j=1, n$ ).

$d_{3 \%}$ of the total reflections were excluded for cross-validation.

doi:10.1371/journal.pone.0036526.t002

$\varepsilon$-amino group of Lys542 (hydrogen bond length $=2.7 \AA$ ). In addition, we observed a contact with the $\varepsilon$-amino group of Lys562 (hydrogen bond length $=3.2 \AA$ ) from the $\beta 6-\beta 7$ strand. Interestingly, the two structures differ with respect to the orientation of the phosphate at position $4(\mathrm{P} 4)$, which contacts either the $\beta 1-\beta 2$ loop or turns $180^{\circ}$ and contacts the $\beta 5-\beta 6$ loop (Figure $2 A, C$ ). In the first case, the main-chain nitrogen of Tyr485 and the carbonyl oxygen of Ser484 (hydrogen bond lengths $=3.0$ and $3.1 \AA$, respectively) of the $\beta 1-\beta 2$ loop region interact with $\mathrm{P} 4$ (Figure $2 \mathrm{~A}$ ), whereas in the other structure the main-chain nitrogen atom of Ser539 of the $\beta 5$ - $\beta 6$ loop forms hydrogen bonds of $2.6 \AA$ with the oxygen atom of $\mathrm{P} 4$ (Figure $2 \mathrm{C}$ ).

The phosphoserine contacts the $\beta 1-\beta 2$ region and the $\beta 5-\beta 6$ loop through the same key residues as $\operatorname{Ins}(4) \mathrm{P}$ (Figure 2D). Specifically, the hydroxyl group of Tyr485 hydrogen bonds with the nitrogen atom of phosphoserine (hydrogen bond length
$=2.9 \AA$ ) and the $\mathrm{NH}_{2}$ group of $\mathrm{Arg} 478$ interacts with the oxygen atom of phosphoserine (hydrogen bond length $=2.8 \AA$ ). In addition, the $\varepsilon$-amino group of Lys542 from the $\beta 5$ - $\beta 6$ loop and the $\varepsilon$-amino group of Lys 562 from the $\beta 7$-strand also interact with the phosphate group of the phosphoserine molecule (hydrogen bond lengths $=2.4 \AA$ and $2.7 \AA$, respectively).

Overall, the ligands show very similar binding modalities and when superposed on each other, they all share common positions in the non-canonical binding site that overlap with previously observed positions of sulfate ions in the holo form of Slm1-PH (Figure 3A). In the non-canonical binding site there are four residues that are consistently involved in ligand binding: Arg478, Tyr485, Lys542 and Lys562. Of these, Arg478 is conserved among PH domains that contain non-canonical binding sites. Interestingly, Arg478 has been shown to be important for ligand binding in vivo by mutation analysis [12]. We subsequently docked 

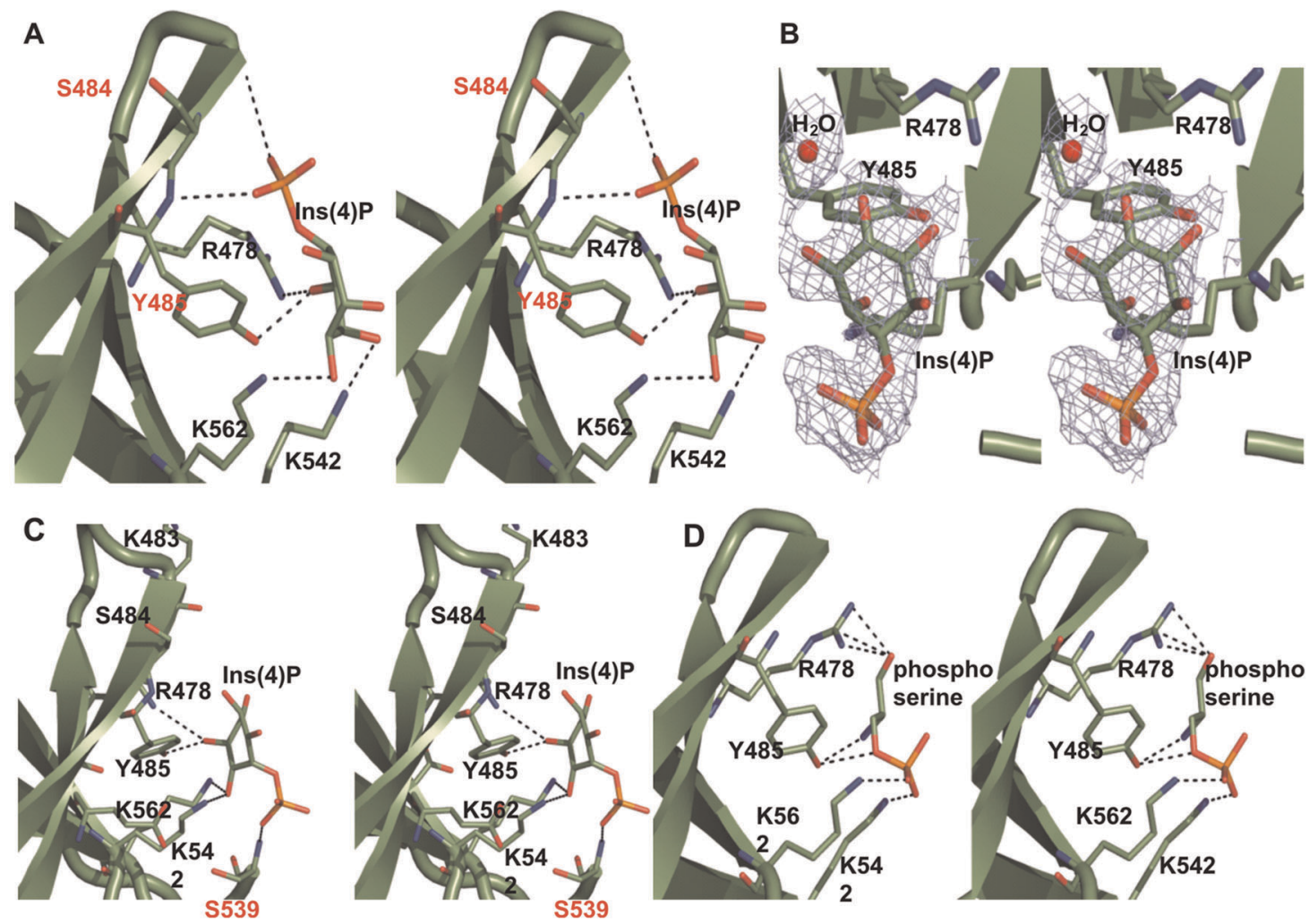

Figure 2. Detailed stereo diagrams of bound ligands. (A) Interactions made by Ins(4)P (green) and the residues at the non-canonical binding site of SIm1-PH (also in green). The phosphate group of Ins(4)P is making contacts with the $\beta 1-\beta 2$ region (residues involved are shown in red). Residues critically involved in the interactions are labeled by the single-letter code and shown in stick representation (see text for details). (B) The Ins(4)P is shown in the experimental 2Fo-Fc electron density map at 1.2 sigma level. The water molecule (red) corresponds to P1 position of Ins $(1,4,5) \mathrm{P}$ when docked into the non-canonical binding site. Position of the Ins(4)P is oriented to maximize the clarity of the figure. (C) Similar interactions are shown here with the Ins(4)P molecule for which the phosphate position is seen on the opposite side of the binding pocket, making contacts with residues of $\beta 5-\beta 6$ (again in red). (D) Stereo representation of phosphoserine bound in the non-canonical binding site. The dashed lines shown between atoms represent hydrogen bonds between ligand(s) and the residues at the non-canonical binding site of SIm1-PH. doi:10.1371/journal.pone.0036526.g002

$\operatorname{Ptd} \operatorname{Ins}(4,5) \mathrm{P}_{2}$ into the non-canonical binding site of Slm1-PH using the structure of $\beta$-spectrin in complex with $\operatorname{Ins}(1,4,5) \mathrm{P}_{3}[18]$. The Ins(4)P and phosphoserine molecules superposed well on to the $\operatorname{Ins}(1,4,5) \mathrm{P}_{3}$ of the $\beta$-spectrin $\mathrm{PH}$ complex positioned in the non-canonical binding site. The resulting model shows that the $\operatorname{Ptd} \operatorname{Ins}(4,5) \mathrm{P}_{2}$ fits well within the binding site, making contact with all conserved residues (Figure 3B). This again suggests a key role for residues Arg478, Tyr485, Lys542 and Lys562 in building a positively charged array that is essential for the correct orientation and anchoring of the $\mathrm{PH}$ domain to $\operatorname{Ptd} \operatorname{Ins}(4,5) \mathrm{P}_{2-}-$ containing membranes. Overall, our results are consistent with the view that the yeast Slm1-PH belongs to the class of PH domains that bind ligands through a non-canonical binding site. Therefore, this type of $\mathrm{PH}$ domain is not restricted to higher eukaryotes, and has been conserved from yeast to mammals.

\section{Potential binding sites for a second anionic lipid head group}

It has been shown that in vitro, Slm1-PH binds to both PtdInsPs $\left(\operatorname{PtdIns}(4) \mathrm{P}, \operatorname{PtdIns}(4,5) \mathrm{P}_{2}\right) \quad[9]$ and DHS-1P in a cooperative manner [12]. We therefore proposed that the Slm1-PH contains both non-canonical and canonical binding sites. Consistent with this hypothesis, our structure here reveals ligand bound in the noncanonical binding site and an additional positively charged region in the canonical binding site that remains available for binding to additional negatively charged head groups (Figure 4A). This encompasses the Arg477, Lys480 and Lys483 residues that point towards the canonical binding site and are important for the recruitment of Slml to biological membranes in vivo [12]. Interestingly, we also observed extra electron densities next to the canonical binding site that could be filled with a phosphate group (Figure 4 and Figure S3A). However, the identity of the ligand could not be unambiguously determined. Crystallization conditions did not contain any phosphate ion but the buffer was mildly acidic ( $\mathrm{pH}$ 6.5), which could lead to partial hydrolysis. This could become significant given the high concentration of ligand used (10-fold excess over Slm1-PH) and the long incubation time (one week) required to obtain optimal crystals. Another possibility is that the full $\operatorname{Ins}(4) \mathrm{P}$ bound, but the electron density for the inositol ring was too poor to be built. Consistent with the latter, we observed that by slightly loosening the restraints, a region of density in continuation with the phosphate group emerged in one out of four molecules in the asymmetric unit. We could fit an inositol ring in this region, even though the densities for some of the hydroxyl groups were missing at 1 sigma level (Figure S3B). This phosphate group lies in the cavity formed by His557, His546, Trp559, Val544 and Phe481 of the neighboring molecule. The oxygen atoms of the phosphate group interact with the $\varepsilon$-amino group of positively charged Lys480 (bond length $=3.2 \AA$ ), $\mathrm{ND}_{1}$ of His557, the $\mathrm{ND}_{2}$ atom of Asn558, and the main-chain nitrogen atom of Asn558 (bond lengths $=2.6 \AA, 2.9 \AA$ and $2.4 \AA$ 
A

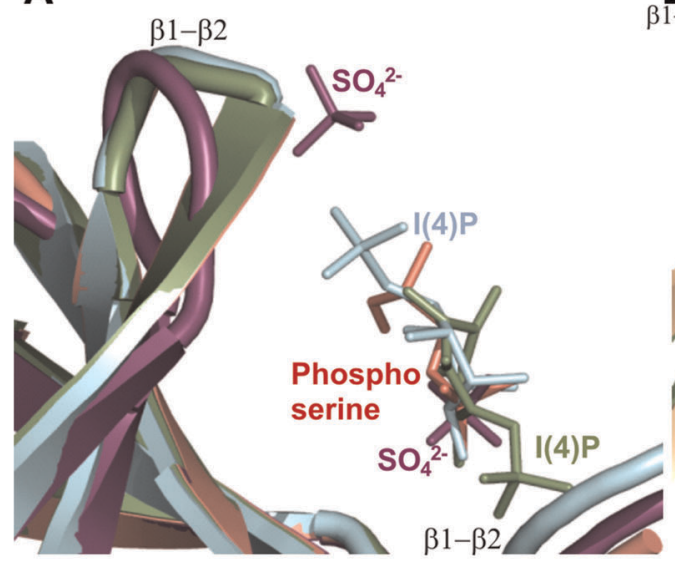

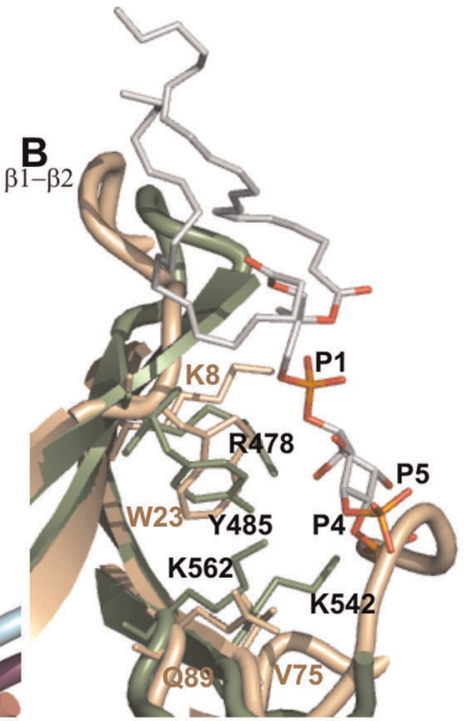

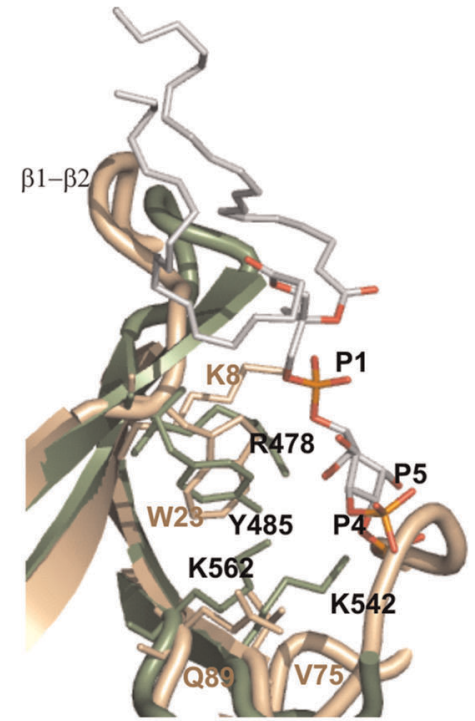

Figure 3. Superposition of SIm1-PH domain structures. (A) The different ligands (sulfate (violet), phosphoserine (salmon), Ins(4)P (green) and also the turned-over Ins(4)P (light blue)) are occupying the same non-canonical binding pocket, confirming the readily available large binding site of the SIm1-PH. (B) Stereo diagram of Ptdlns $(4,5) \mathrm{P}_{2}$ (atom colors) modeled in the non-canonical binding site of SIm1-PH (green) and overlaid onto the $\beta$ spectrin PH domain (wheat color). This shows the conservation of side chains that contact the ligands. doi:10.1371/journal.pone.0036526.g003

respectively) through hydrogen bonds (Figure S3A). We observed that the long and flexible side-chain of Arg477 points towards the phosphate group (bond length $=8.5 \AA$ ) (Figure $4 \mathrm{~B}$ ) and the two could be modeled as close as $3 \AA$ (Figure S3C). Our structures show that, along with other residues, Arg477 and Arg478 contribute to ligand binding in both of the two putative binding sites (Figure 4B and Figure S4). This is consistent with previous analyses, which showed that mutation of these residues to alanine either alone or in combination caused defects in Slml function and its ability to associate with biological membranes ([12] and Figure S4). The stronger and apparently cumulative effect of the double mutant supports the view that binding to both sites is required for Slml function. Taken together, our results clearly suggest the existence of an additional positively charged binding region adjacent to the non-canonical binding site.

Comparison of SIm1-PH domain with other $\mathrm{PH}$ domains Several structures of different $\mathrm{PH}$ domains have been determined using X-ray crystallography or nuclear magnetic resonance (NMR) spectroscopy [18,27,28,29,30]. Many of these are in complex with inositol phosphate ligand bound in either the canonical or the non-canonical binding site (Figure 5). Irrespective
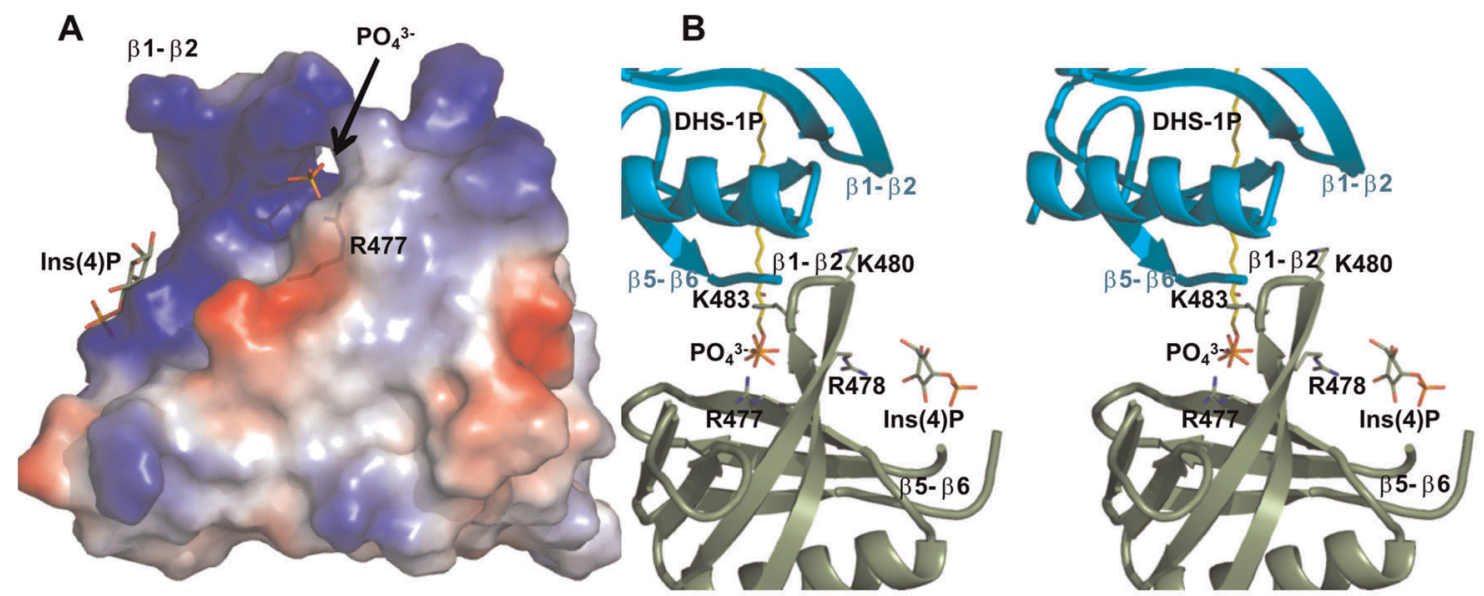

Figure 4. Surface charge distribution. (A) The SIm1-PH provides a positively-charged cavity to interact with a negatively charged Ins(4)P molecule (in green). The back of the $\beta 1-\beta 2$ region (by rotating SIm1-PH by 180 degrees) shows a more positively charged region. In several structures of SIm1-PH determined in complex with ligand analogs, we built a phosphate group or full Ins(4)P at this additional positively charged site (see text for details). The Arg477 side chain that turns towards the canonical binding site is also shown in transparent stick format. (B) Stereo diagram of Slm1$\mathrm{PH}$ (green) showing Arg477, Arg478, Lys480 and Lys483 on either side of the $\beta 1-\beta 2$ strands in the vicinity of the negatively charged residues of the $\beta 5-\beta 6$ loop of the neighboring molecule (cyan). Part of the $\beta 5-\beta 6$ loop is truncated for the clarity of the figure. The bound phosphate group is shown in stick representation at the back of the $\beta 1-\beta 2$ region towards the canonical binding site. We have modeled the natural ligand (DHS-1P in yellow) aligning with the phosphate position in this region. Ins(4)P is also shown in the non-canonical binding site.

doi:10.1371/journal.pone.0036526.g004 

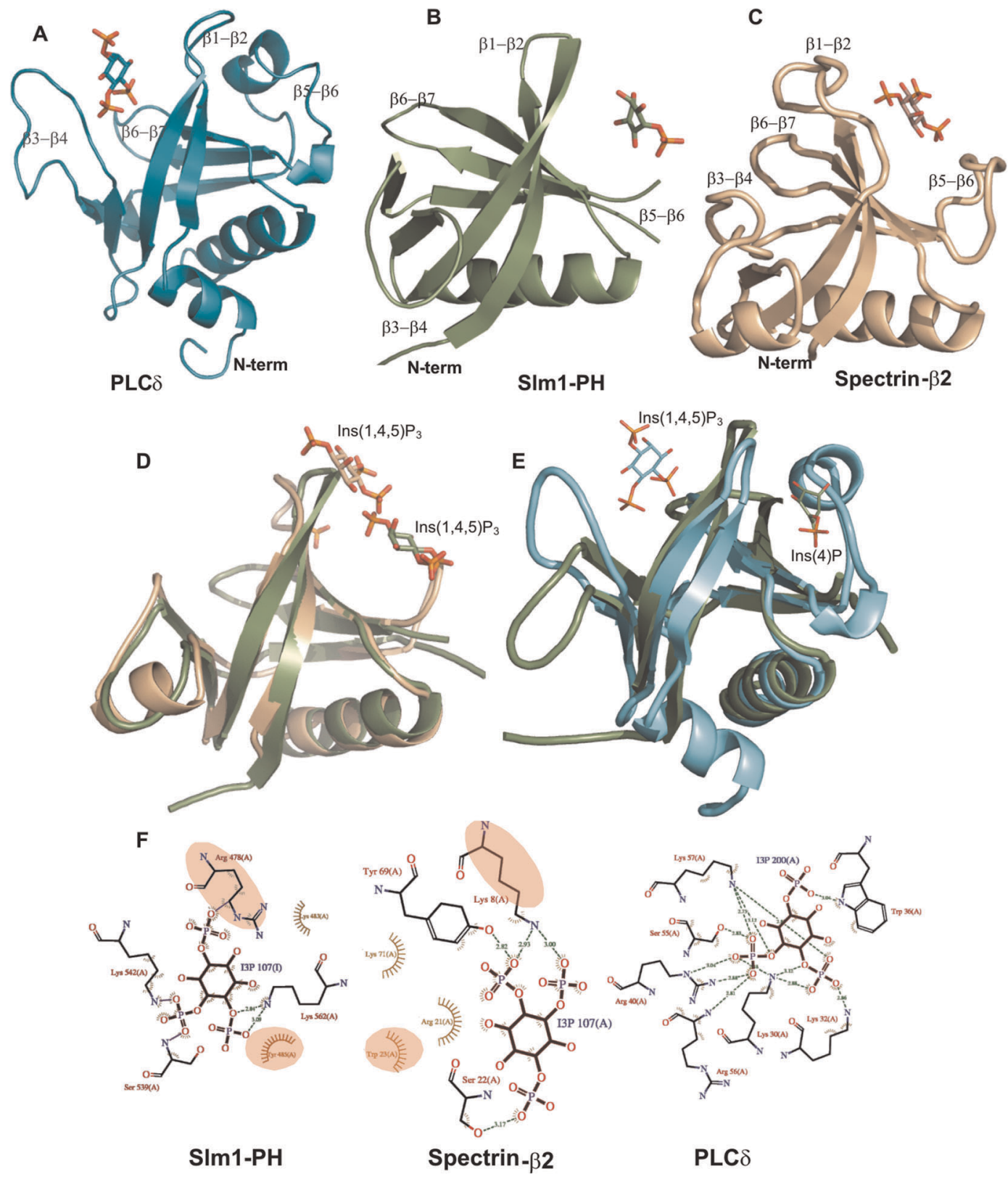

Figure 5. Comparison of canonical and non-canonical binding sites. (A) Structure of the PLC $\delta$ (1MAI.PDB) in steel blue with bound Ins $(1,4,5) \mathrm{P}_{3}$ in the canonical binding site, which is formed by $\beta 1-\beta 2, \beta 3-\beta 4$ and $\beta 6-\beta 7$ loop regions; (B) Cartoon representation of SIm1-PH in green, Ins(4)P is bound in the non-canonical binding site; (C) Structure of the $\beta$-spectrin PH domain (1BTN.PDB) in wheat color with bound Ins $(1,4,5) \mathrm{P}_{3}$ again in the non-canonical binding site. (D) Superposition of the SIm1-PH (green) modeled with $\operatorname{Ins}(1,4,5) \mathrm{P}_{3}$ onto the $\beta$-spectrin PH domain (wheat) and (E) onto PLC $\delta$ (cyan) (F) LIGPLOT representation [38] showing residues involved in ligand binding in the non-canonical binding site (SIm1-PH and $\beta$ spectrin) and the canonical binding site (PLC 8 ). The conserved residues between PH domains of $\operatorname{SIm} 1$ and $\beta$-spectrin involved in ligand binding are highlighted in pale yellow.

doi:10.1371/journal.pone.0036526.g005

of the binding scenario (canonical or non-canonical), a series of positively charged residues within the $\beta 1-\beta 2$ loop region are relatively conserved (Figure 6; black box). A structure-based sequence alignment with other $\mathrm{PH}$ domains reveals that Slm1-PH is most similar to the $\mathrm{PH}$ domains of $\beta$-spectrin, Tiaml and ArhGAP9, which bind to ligands through their non-canonical sites. The superposition of Slm1-PH onto the PH domain of $\beta$ spectrin shows the conserved $\beta 1-\beta 2$ loop region accommodating the ligand in the non-canonical binding site (Figure 5D and F). Indeed, Slm1-PH lacks the characteristic KXK/RXR motif in the $\beta 1-\beta 2$ loop that is present in $\mathrm{PH}$ domains with a canonical binding site (b/akt, Btk, Grpl and PLC $\delta[5,6,8,18,19]$ (Figure 5E and F). Also in Slml-PH, the most conserved positively charged residue within the $\beta 1-\beta 2$ loop ( $\operatorname{Arg} 478)$ is flipped towards the noncanonical binding site to interact with the $\operatorname{Ins}(4) \mathrm{P}$ molecule. In PLC $\delta$, a small valine residue occupies a similar position and also points towards the non-canonical binding site. This is consistent with the fact that the space between the $\beta 1-\beta 2$ and the $\beta 5-\beta 6$ loops is very narrow, which excludes the non-canonical binding mode in PLC $\delta$ (Figure S4). 


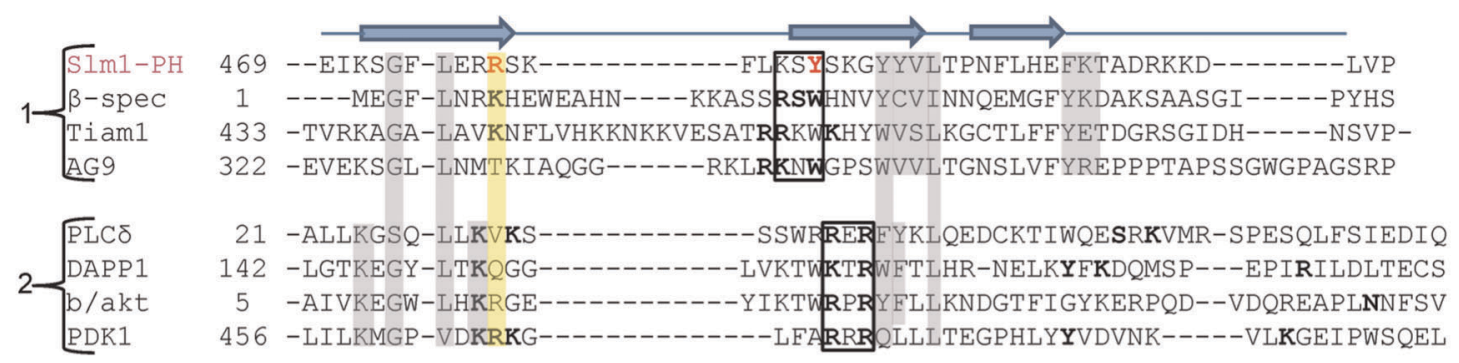

$1\left\{\begin{array}{l}\text { SIm1-PH } \\ \beta-\text { spec } \\ \text { Tiam1 } \\ \text { AG9 }\end{array}\right.$
$2\left\{\begin{array}{l}\text { PLC } \delta \\ \text { DAPP1 } \\ \text { /akt } \\ \text { PDK1 }\end{array}\right.$

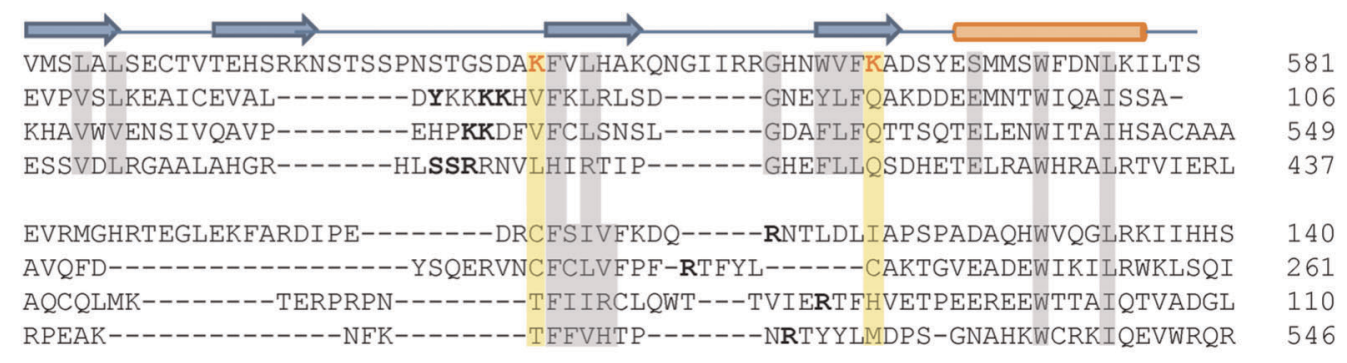

Figure 6. Structure-based sequence alignment of PH domains of SIm1, $\boldsymbol{\beta}$-spectrin and PLC $\boldsymbol{\delta}$. The alignment was produced using the PROMALS3D program [39] and corrected manually on the basis of the three-dimensional structure of the Slm1-PH. The matched-up sequences were from RCSB/PDB. The secondary structure elements revealed in the SIm1-PH crystal structure are shown above the sequence: $\beta$-sheets are shown by blue arrows and the C-terminal helix by an orange cylinder. The residues of SIm1-PH involved in Ins(4)P interaction are shown in red. The residues highlighted in yellow are aligned with the ligand-interacting residues of the SIm1-PH. Residues of different classes of PH domains that are involved in ligand binding are shown in 'bold'. The R/KXR and R/KXW motifs for ligand binding in the canonical and non-canonical binding sites respectively, are shown in the black box. The structure-based sequence alignment showing overall conserved or semi-conserved residues are represented in grey. Sequences are grouped according to ligand binding in the non-canonical and canonical binding pockets. doi:10.1371/journal.pone.0036526.g006

Similar to $\beta$-spectrin, Tiaml and ArhGAP9, the $\beta 1-\beta 2$ loop of Slm1-PH contains a conserved R/KXW motif, which is involved in ligand binding at the non-canonical binding site. The only minor difference is that the tryptophan is replaced by another aromatic residue, Tyr485. We also observe interesting differences between Slm1-PH and $\beta$-spectrin, Tiam1 and ArhGAP9 that may account for the dual binding specificity of Slm1-PH. For example, a non-conserved positively charged residue (Lys542) participates in ligand binding in the Slm $1 \mathrm{PH}$, whereas a series of positively charged residues are absent within the $\beta 5-\beta 6$ loop region. Finally, in the case of Slm 1-PH, ligand binding is also supported by Lys562 of the $\beta 6-\beta 7$ region (Figure 6 ), which is not conserved in other $\mathrm{PH}$ domains. These differences are consistent with the hypothesis that Slm1-PH may have altered binding properties compared with previously characterized $\mathrm{PH}$ domains.

In summary, here we present the first three-dimensional structures of the $\operatorname{Slm} 1-\mathrm{PH}$ bound to $\operatorname{Ins}(4) \mathrm{P}$ and phosphoserine and show that the Slml-PH exhibits a non-canonical PtdInsPsbinding mechanism. The non-canonical binding site is shallow, large and solvent-exposed, and so the phosphate position of Ins(4)P in one of the structures was seen on the opposite side of the molecule. The non-canonical binding site accommodated anions from the crystallization conditions, and also the phosphoserine molecule. The long $\beta 5-\beta 6$ loop occupied part of the space at the canonical binding cavity, making it less available for ligand binding. We nevertheless observed a large positively charged region behind the $\beta 1-\beta 2$ loop towards the canonical binding site that associated with a phosphate group. This putative binding surface could accommodate a second ligand and account for the cooperative binding described for the SIm1-PH.

\section{Materials and Methods}

\section{Expression, purification and crystallization}

The Slm1-PH was expressed from Escherichia coli and purified using affinity chromatography. Cloning, expression and purification of the recombinant protein used in this study were performed using the methods described previously [12].

\section{Crystallization of the SIm1-PH domain}

Initial apo crystals of the Slml-PH were obtained through a screen with the mosquito crystallization robot (TTP Labtech) using the sitting drop vapor diffusion technique in 96-well plates with drop and reservoir volumes of $0.2 \mu \mathrm{l}$ and $100 \mu \mathrm{l}$, respectively. Crystals were routinely grown in 24-well plates (Hampton Research) at $15^{\circ} \mathrm{C}$ by the hanging drop vapor diffusion technique. Equal volumes $(0.75 \mu \mathrm{l} / 0.75 \mu \mathrm{l})$ of protein $(15 \mathrm{mg} / \mathrm{ml})$ and crystallization buffer $(0.1 \mathrm{M}$ MES + 30\% PEG5000 + $0.2 \mathrm{M}$ ammonium sulfates, $\mathrm{pH}$ 6.5) were mixed on a cover slip, which was subsequently equilibrated against a reservoir containing $500 \mu \mathrm{l}$ of crystallization buffer. Micro-seeding was used to obtain larger single crystals. The optimal hexagonal crystals grew in about eight days to a size of approximately $100 \mu \mathrm{m} \times 100 \mu \mathrm{m} \times$ $50 \mu \mathrm{m}$. Subsequently, sulfate ions were replaced with malonate in the crystallization conditions (0.1 M MES + 28\% PEG5000 + $0.2 \mathrm{M}$ sodium malonate, $\mathrm{pH} 6.5$ ) to avoid interference with ligand binding.

Co-crystallization and soaking techniques were used to obtain complex crystals. Several crystals of Slm1-PH were obtained with and without head group analogues of PtdInsPs and lipids (Table 1). Crystals of Slm1-PH in complex with Ins(4)P were obtained by using 10-fold molar excess of Ins(4)P (Sigma Aldrich). Monoclinic rod-like crystals formed in about a week and grew to approximately $150 \mu \mathrm{m} \times 90 \mu \mathrm{m}$ × $300 \mu \mathrm{m}$ from the crystallization 
conditions containing $0.1 \mathrm{M}$ Bis-Tris $+25 \%$ PEG3350 + $0.2 \mathrm{M}$ sodium malonate, $\mathrm{pH}$ 5.5. All crystals were harvested by transfer to a cryo-protectant solution of $15 \%$ ethylene glycol in the mother liquor, rapidly collected in a nylon loop (Hampton Research, USA) and flash-cooled in liquid nitrogen.

\section{Data collection, structure determination and refinement}

Data were collected at various synchrotron beamlines at the European Synchrotron Radiation Facility (ESRF) in Grenoble, France and the Swiss Light Source (SLS) in Villigen, Switzerland. All the data sets were processed and scaled using the XDS program package [31]. For all the structures presented here, the phase problem was solved by molecular replacement [32] using the program PHASER [33] and the coordinates of apo Slm1-PH (3NSU; [12]) as a search model. Two flexible loops (residues: 501512 and 526-542) of the search model were truncated for the phase solution. The model was refined in alternating cycles of refinement with PHENIX [34]. Manual rebuilding and visual comparisons were made using the graphical program COOT [35].

The stereo chemical quality of the final model was assessed using PROCHECK [36]. Molecular diagrams were drawn using the PyMOL molecular graphics suite [37]. Details of the diffraction data collection, quality and refinement statistics are given in Table 2.

\section{Protein databank accession number}

The atomic coordinates and structure factor amplitudes have been deposited in the Protein Data Bank Europe, http://www.ebi. ac.uk/pdbe/ [PDB ID codes: 4A5K, 4A6F, 4A6H and 4A6K].

\section{Supporting Information}

Figure S1 Chemical drawings of the physiological ligands of the Slm1-PH. The head-group mimics, Ins(4)P and phosphoserine, are indicated by red circles.

(TIF)

Figure S2 Experimental Fo-Fc electron density map prior to modeling of a bound Ins $(4) P$ molecule, contoured at 2.5 sigma level. Ins(4)P molecule and the residues involved in the interaction are shown in the stick format and labeled accordingly.

(TIF)

\section{References}

1. Ferguson KM, Lemmon MA, Sigler PB, Schlessinger J (1995) Scratching the surface with the PH domain. Nat Struct Biol 2: 715-718.

2. Lemmon MA, Ferguson KM (2000) Signal-dependent membrane targeting by pleckstrin homology $(\mathrm{PH})$ domains. Biochem J 350 Pt 1: 1-18.

3. Byrne RD, Rosivatz E, Parsons M, Larijani B, Parker PJ, et al. (2007) Differential activation of the PI 3-kinase effectors AKT/PKB and p70 S6 kinase by compound 48/80 is mediated by PKCalpha. Cell Signal 19: 321-329.

4. Calleja V, Laguerre M, Parker PJ, Larijani B (2009) Role of a novel PH-kinase domain interface in $\mathrm{PKB} / \mathrm{Akt}$ regulation: structural mechanism for allosteric inhibition. PLoS Biol 7: e17.

5. Ferguson KM, Kavran JM, Sankaran VG, Fournier E, Isakoff SJ, et al. (2000) Structural basis for discrimination of 3-phosphoinositides by pleckstrin homology domains. Mol Cell 6: 373-384.

6. Ferguson KM, Lemmon MA, Schlessinger J, Sigler PB (1995) Structure of the high affinity complex of inositol trisphosphate with a phospholipase C pleckstrin homology domain. Cell 83: 1037-1046.

7. Harlan JE, Hajduk PJ, Yoon HS, Fesik SW (1994) Pleckstrin homology domains bind to phosphatidylinositol-4,5-bisphosphate. Nature 371: 168-170.

8. Thomas CG, Deak M, Alessi DR, van Aalten DM (2002) High-resolution structure of the pleckstrin homology domain of protein kinase b/akt bound to phosphatidylinositol (3,4,5)-trisphosphate. Curr Biol 12: 1256-1262.

9. Audhya A, Loewith R, Parsons AB, Gao L, Tabuchi M, et al. (2004) Genomewide lethality screen identifies new PI4,5P2 effectors that regulate the actin cytoskeleton. EMBO J 23: 3747-3757.
Figure S3 Phosphate positioned in the cavity at the back of the $\boldsymbol{\beta} 1-\boldsymbol{\beta} 2$ region. (A) Interaction with the residues of the $\beta 1$ $\beta 2$ and $\beta 6-\beta 7$ loops. The phosphate group and the residues involved in the interaction are shown in ball-and-stick format in the ribbon diagram of Slm1-PH in green. All the hydrogen bonds are shown by dashed lines. (B) Cartoon representation of Slm 1-PH with $\operatorname{Ins}(4) \mathrm{P}$ bound at the back of the $\beta 1-\beta 2$ loop region in one out of four molecules in the asymmetric unit. $(\mathbf{G})$ Here we modeled/ rotated the side chain of Arg477 (already facing towards the phosphate of $\operatorname{Ins}(4) \mathrm{P})$ that can come to within $3 \AA$ of the phosphate group.

(TIF)

Figure S4 Superposition of the conserved Arg477 and Arg478 of Slm1-PH (green) onto the residues Lys and Val of PLC $\delta$ (teal color) and Arg and Lys of $\beta$-spectrin (wheat color) PH domains. (B) Impact of Arg477 and Arg478 mutation to alanine on Slm 1 recruitment to biological membranes and Slm 1 function in vivo, i.e. yeast growth and actin polarization. Adapted from [12].

(TIF)

\section{Acknowledgments}

This study was performed at EMBL Heidelberg (EMBL-HD). The authors thank the beam line staff at the European Synchrotron Radiation Facility (ESRF), beam line ID23-1, Grenoble, France and the Swiss Light Source (SLS) at Villigen, Switzerland, where all the data collections were performed. The authors thank O. Barabas for careful reading of the manuscript and critical comments. We thank H. Besir from the protein purification facility at EMBL-HD for protein purification. We thank A. Scholz and C. Mueller's group for the crystallization trials on the mosquito robot at EMBL-HD. We thank the colleagues from the Structural and Computational Biology Unit and Graffinity Pharmaceutical GmbH for providing inspiring and fruitful discussions and O. Gallego for his participation. We also thank T. Stuwe and A. Ladurner for collecting some of the data set at the ESRF, Grenoble synchrotron station.

\section{Author Contributions}

Conceived and designed the experiments: KA ACG. Performed the experiments: KA. Analyzed the data: KA KM ACG. Contributed reagents/materials/analysis tools: KA ACG. Wrote the paper: KA ACG. Contribution type: KA ACG.

10. Daquinag A, Fadri M, Jung SY, Qin J, Kunz J (2007) The yeast PH domain proteins Slm 1 and Slm2 are targets of sphingolipid signaling during the response to heat stress. Mol Cell Biol 27: 633-650.

11. Saraste M, Hyvonen M (1995) Pleckstrin homology domains: a fact file. Curr Opin Struct Biol 5: 403-408.

12. Gallego O, Betts MJ, Gvozdenovic-Jeremic J, Maeda K, Matetzki C, et al. (2010) A systematic screen for protein-lipid interactions in Saccharomyces cerevisiae. Mol Syst Biol 6: 430.

13. Lemmon MA (2004) Pleckstrin homology domains: not just for phosphoinositides. Biochem Soc Trans 32: 707-711.

14. Parker PJ (1999) Inhibition of protein kinase C - do we, can we, and should we? Pharmacol Ther 82: 263-267.

15. Parker PJ, Hemmings BA, Gierschik P (1994) PH domains and phospholipases a meaningful relationship? Trends Biochem Sci 19: 54-55.

16. Maffucci T, Falasca M (2001) Specificity in pleckstrin homology (PH) domain membrane targeting: a role for a phosphoinositide-protein co-operative mechanism. FEBS Lett 506: 173-179.

17. Ceccarelli DF, Blasutig IM, Goudreault M, Li Z, Ruston J, et al. (2007) Noncanonical interaction of phosphoinositides with pleckstrin homology domains of Tiam1 and ArhGAP9. J Biol Chem 282: 13864-13874.

18. Hyvonen M, Macias MJ, Nilges M, Oschkinat H, Saraste M, et al. (1995) Structure of the binding site for inositol phosphates in a PH domain. EMBO J 14: 4676-4685. 
19. Hyvonen M, Saraste M (1997) Structure of the PH domain and Btk motif from Bruton's tyrosine kinase: molecular explanations for X-linked agammaglobulinaemia. EMBO J 16: 3396-3404.

20. Fadri M, Daquinag A, Wang S, Xue T, Kunz J (2005) The pleckstrin homology domain proteins Slm 1 and SIm2 are required for actin cytoskeleton organization in yeast and bind phosphatidylinositol-4,5-bisphosphate and TORC2. Mol Biol Cell 16: 1883-1900.

21. Terawaki S, Kitano K, Mori T, Zhai Y, Higuchi Y, et al. (2010) The PHCCEx domain of Tiam $1 / 2$ is a novel protein- and membrane-binding module. EMBOJ 29: $236-250$.

22. Mansoor M, Melendez AJ (2008) Recent trials for FTY720 (fingolimod): a new generation of immunomodulators structurally similar to sphingosine. Rev Recent Clin Trials 3: 62-69.

23. Zhang Z, Schluesener HJ (2007) FTY720: a most promising immunosuppressant modulating immune cell functions. Mini Rev Med Chem 7: 845-850.

24. Hiestand PC, Rausch M, Meier DP, Foster CA (2008) Ascomycete derivative to MS therapeutic: S1P receptor modulator FTY720. Prog Drug Res 66: 361, 363381.

25. Isakoff SJ, Cardozo T, Andreev J, Li Z, Ferguson KM, et al. (1998) Identification and analysis of PH domain-containing targets of phosphatidylinositol 3-kinase using a novel in vivo assay in yeast. EMBO J 17: 5374-5387.

26. Edlich C, Stier G, Simon B, Sattler M, Muhle-Goll G (2005) Structure and phosphatidylinositol- $(3,4)$-bisphosphate binding of the C-terminal $\mathrm{PH}$ domain of human pleckstrin. Structure 13: 277-286.

27. Rameh LE, Arvidsson A, Carraway KL 3rd, Couvillon AD, Rathbun G, et al. (1997) A comparative analysis of the phosphoinositide binding specificity of pleckstrin homology domains. J Biol Chem 272: 22059-22066.

28. Kavran JM, Klein DE, Lee A, Falasca M, Isakoff SJ, et al. (1998) Specificity and promiscuity in phosphoinositide binding by pleckstrin homology domains. J Biol Chem 273: 30497-30508.
29. Koshiba S, Kigawa T, Kim JH, Shirouzu M, Bowtell D, et al. (1997) The solution structure of the pleckstrin homology domain of mouse Son-of-sevenless 1 (mSos 1). J Mol Biol 269: 579-591.

30. Yu JW, Mendrola JM, Audhya A, Singh S, Keleti D, et al. (2004) Genome-wide analysis of membrane targeting by $\mathrm{S}$. cerevisiae pleckstrin homology domains. Mol Cell 13: 677-688.

31. Kabsch W (1993) Automatic processing of rotation diffraction data from crystals of initially unknown symmetry and cell constants. Journal of Applied Crystallography 26: 795-800.

32. Rossmann MG (1990) The molecular replacement method. Acta Crystallogr A 46 (Pt 2): 73-82.

33. Read RJ (2001) Pushing the boundaries of molecular replacement with maximum likelihood. Acta Crystallographica Section D-Biological Crystallography 57: 1373-1382.

34. Adams PD, Grosse-Kunstleve RW, Hung LW, Ioerger TR, McCoy AJ, et al. (2002) PHENIX: building new software for automated crystallographic structure determination. Acta Crystallogr D Biol Crystallogr 58: 1948-1954.

35. Emsley P, Cowtan K (2004) COOT: model-building tools for molecular graphics. Acta Crystallogr D60: 2126-2132.

36. Laskowski RA, MacArthur MW, Moss DS, Thornton JM (1993) PROCHECK: a program to check the stereochemical quality of protein structures. J Appl Crystallogr 26: 283-291

37. DeLano WL (2002) The PyMOL Molecular Graphics System. In. San Carlos CA, USA: DeLano Scientific; Pymol website. Available: http://www.pymol. org/. Accessed 2012 Jan 27.

38. Wallace AC, Laskowski RA, Thornton JM (1995) LIGPLOT: a program to generate schematic diagrams of protein-ligand interactions. Protein Eng 8: 127-134.

39. Pei J, Kim BH, Grishin NV (2008) PROMALS3D: a tool for multiple protein sequence and structure alignments. Nucleic Acids Res 36: 2295-2300. 